\title{
RECENT DEVELOPMENTS OF THE EXCYT PROJECT
}

\author{
G. Ciavola, R. Alba, L. Barone Tonghi, L. Calabretta, L. Celona, G. Cuttone, G. Di Bartolo, \\ P. Finocchiaro, S. Gammino, M. Menna, R. Papaleo, G. Raia, D. Rifuggiato, A. Rovelli, \\ M. Silvestri, D. Vinciguerra, M. Winkler, H. Wollnik
}

INFN - Laboratori Nazionali del Sud, Via S. Sofia 44, 95123 Catania, Italy

\begin{abstract}
The EXCYT project at LNS, Catania, Italy aims to the production of radioactive ion beams to be accelerated by a tandem up to $8 \mathrm{MeV} / \mathrm{amu}$. The primary beam coming from an ECR ion source goes into a K800 cyclotron and then to the target-ion source assembly. Here the species of interest are produced mainly by projectile fragmentation in a target thicker than the projectile range. The products diffuse through the heated target, are desorbed from its surface and go to the ion source via a transfer line. After ionization, the products are mass-separated and injected into the tandem to be post-accelerated and delivered to the users. It is worth noting that besides the post acceleration there is also an option to supply $300 \mathrm{keV}$ beams for low energy experiments. In the following, the status of the project will be described into details.
\end{abstract}

\section{ECR ION SOURCES AND SUPERCONDUCTING CYCLOTRON}

In 1998 the superconducting ECR (electron cyclotron resonance) ion source SERSE has been installed. The source has already achieved the maximum primary beam intensities required by the EXCYT project $(7 \mathrm{p} \mu \mathrm{A}$ of totally stripped light ions) [1]. To ensure the continuous operation of the cyclotron, a second ECR ion source with room-temperature magnets has been recently added.

The K800 Superconducting Cyclotron (CS) is presently operated as a booster for the $15 \mathrm{MV}$ tandem beam [2]. To allow its future use in a stand-alone mode and to obtain the beam intensities needed, axial injection has been chosen. The axial injection line from the two ECR ion sources to the CS is being completed; it will allow the rotation of the beam ellipse in the final matching point (MP). The remaining vertical section has been recently designed to achieve a small beam size at the entrance of the inflector [2] . For the new injection mode an inflector and a second harmonic central region (fig.1) will replace the present stripper system. To operate the cyclotron in a constant orbit mode the source, the dees and the inflector voltages will be varied according to the relation: $\mathrm{V} /\left(\omega_{0} \mathrm{~B}_{\mathrm{o}}\right)=$ const., with a source limiting voltage of $30 \mathrm{kV}$ [3]. The spiral inflector has a $6 \mathrm{~mm}$ gap and an applied electric field of $22 \mathrm{kV} / \mathrm{cm}$ : the latter value has been chosen to make the exit radius as large as possible (17.5 $\mathrm{mm}$ for $30 \mathrm{kV}$ injection voltage) for comfortably allocating the central region posts. The design of the central region is based upon the MSU K500 cyclotron [4], which has been refurbished to be coupled to the K1200 cyclotron. At MSU, the whole system is conceived to deliver intense primary beams for the production of radioactive ion beams by fragmentation. The puller, consisting of two posts at the RF voltage immediately accelerates the beam coming out of the inflector. The particular geometry of the RF and ground posts allows the particles to gain as much energy as possible. The beam is well confined inside the central region, while there is a radial dependence upon the phase at the end of the first turn. Here the last ground slit of the central region performs a rough phase selection, reducing the phase range down to approximately $35^{\circ}$ (from $200^{\circ}$ to $235^{\circ}$ ) which corresponds to the acceptance of the cyclotron.

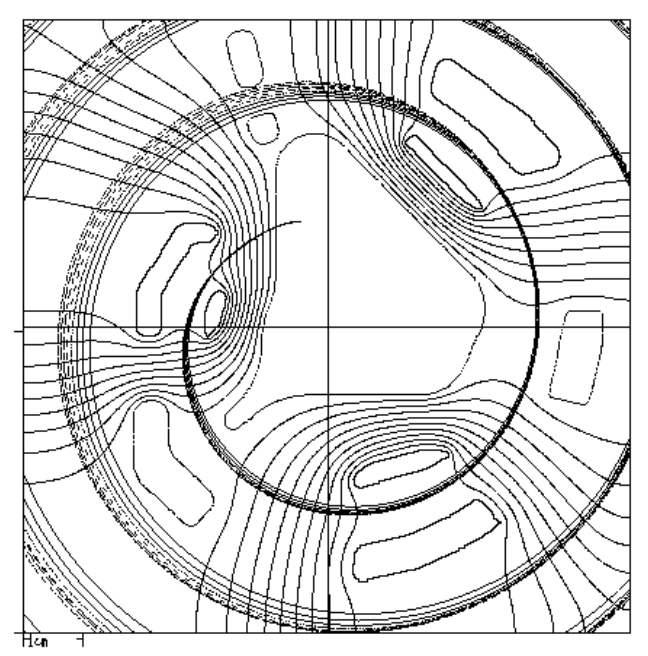

Figure 1: The $2^{\text {nd }}$ harmonic central region of the LNS Superconducting Cyclotron. Central trajectories with starting times from $200^{\circ}$ to $235^{\circ}$ are plotted.

A slit system [2] has been designed to perform a fine phase selection out of the central region: it consists of three wedges that will discriminate among phases according to the simulations made in [5]. The matching between the line and the cyclotron in the new mode has been evaluated with good result [3] by considering separately: a) the beam ellipse transport from the MP to 
the inflector exit and b) the evolution of the beam ellipse from the inflector exit towards the extraction. The central region parts and the inflector are planned to be installed in the cyclotron by November 1999 .

\section{HV-PLATFORMS}

Two different $250 \mathrm{kV}$ platforms will be built in separated rooms. The voltage has been chosen in order to enhance both the injection in the tandem and the performance of the mass separator. Most of the radioactivity is generated and confined within the first platform area housing the target-ion source assembly and the pre-separator; the whole area is contained in a shielded pit. The radioactivity level in the second platform area is much lower; this platform houses the charge exchange cell and the first stage of the isobaric mass separator. A high-voltage conduit carrying the beam line and the electrical cables through a 1-meter thick shield wall connects the two platforms. The conduit consists of two concentric pipes: a continuous electrically conductive inner pipe and a continuous epoxy outer pipe with flanges that extend into the $250 \mathrm{kV}$ platforms by a few centimetres. The space between the two pipes is filled with 2 atm of $\mathrm{SF}_{6}$ insulating gas. Moreover, within the span of the shield wall a stainless steel tube with field termination rings covers the epoxy tube. POISSON simulations showed that a) the electrical gradients are appropriate to prevent sparks; b) for a $250 \mathrm{kV}$ platform voltage the maximum value of the electric field is located at both ends of the stainless steel tube and reaches up to $13 \mathrm{kV} / \mathrm{cm}$.

\section{TARGET-ION SOURCE ASSEMBLY}

Several innovative high-power target geometries have been developed and are currently being constructed. The designs are based on the concept of redistributing the primary beam intensity over larger transverse and longitudinal target dimensions. At operating temperatures above $\sim 2000{ }^{\circ} \mathrm{C}$ radiative cooling is the dominant heat transfer process: therefore geometrical target configurations which allow maximum viewing of cool surfaces will radiate most efficiently. The surface is increased by tilting the target of an angle $\alpha$ (Fig.2) chosen to reach the desired level of temperature. By wiggling or defocusing the beam, its spot size on the target should be increased up to $\sim 30 \mathrm{~mm}$, thus effectively reducing the beam power density. The combined effect of increasing both the beam size and the target dimensions will increase the effective radiating surface area of the latter, thus resulting in a stable target operation with increased total beam intensities. Computer simulations by ANSYS finiteelement code, validated this concept which will be also used for the HRIBF at ORNL [6]. A special type of graphite with high open porosity has been chosen for the initial targets: this material should allow a good diffusion of the products.

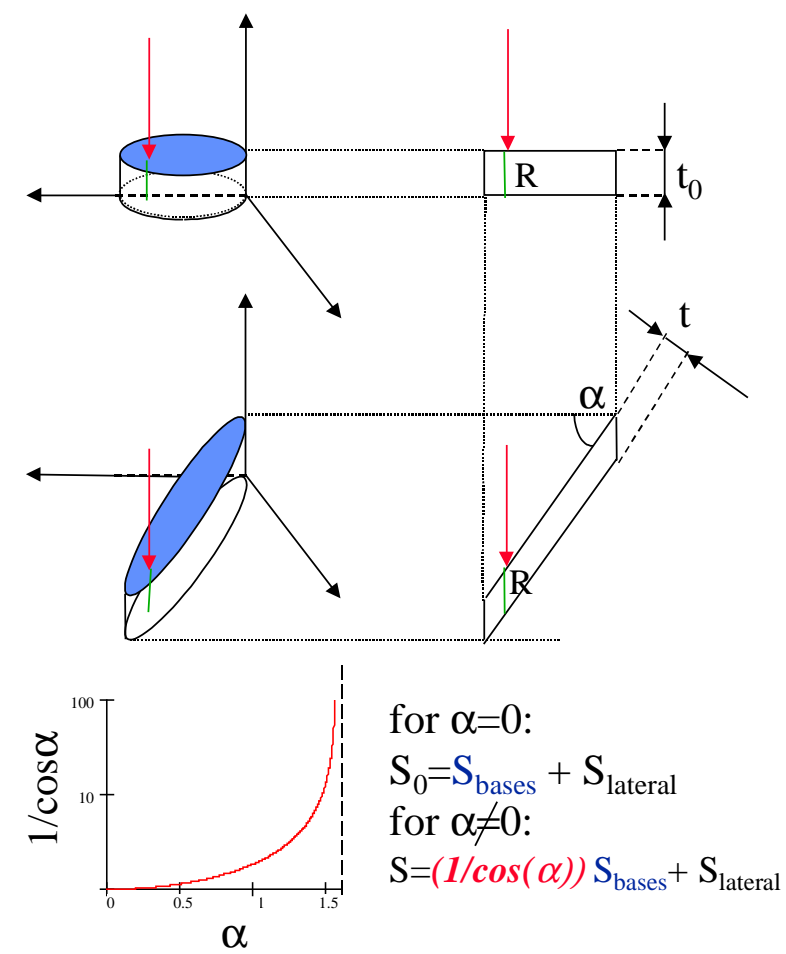

Figure 2: The scheme of the EXCYT tilted target of the Project. Assuming $R$ as the range of each particle equal to $t_{0}$, for any $\alpha$ value the particle will reach the bottom of the target. By increasing $\alpha$, the target thickness increases as well as the surface area following the shown relation.

Three ISOLDE-type ion sources, namely positive surface ionisation, negative surface ionisation and hot plasma, are ready to be tested. A fourth negative ion source, developed for fluorine at the HRIBF will be built and tested for EXCYT. The microwave discharge ion source MIDAS has been completed in 1997. Gas efficiency tests showed fast ionization times (a few $\mathrm{ms}$ ) and the almost complete independence on the ionic species. On the other hand, they also showed the inadequacy of using an antenna-based injection system. The source has been redesigned with a waveguide injection and a better extraction system. If the expected enhancement (up to $20 \%$ ) will be achieved by the second prototype, a final version finely matching both with the target and with the front end will be designed. The ground electrode has been designed with an extraction optics able to interact with all of the above mentioned ion sources.

A massive experimental programme has been undertaken in order to estimate the formation of products inside the targets, their release by means of diffusion and desorption and finally the efficiencies of the ion sources. Cross sections for several nuclides produced by the reaction: ${ }^{19} \mathrm{~F}$ $(50 \mathrm{MeV} / \mathrm{amu})+{ }^{12} \mathrm{C}$ have been measured, with values: 91 $\mathrm{mb}$ for ${ }^{7} \mathrm{Be}, 96 \mathrm{mb}$ for ${ }^{18} \mathrm{~F}, 10 \mathrm{mb}$ for ${ }^{22} \mathrm{Na}, 2.5 \mathrm{mb}$ for ${ }^{24} \mathrm{Na}$ [7]. The figures for ${ }^{18} \mathrm{~F}$ and ${ }^{7} \mathrm{Be}$ are very promising and can be increased with higher energy beams (e.g. 80 $\mathrm{MeV} / \mathrm{amu}$ ). Using ${ }^{9} \mathrm{Be}$ as a projectile on graphite should 
also increase the latter value. Experiments to determine the cross section for ${ }^{9} \mathrm{Be}(50 \mathrm{MeV} / \mathrm{amu})+{ }^{12} \mathrm{C} \rightarrow{ }^{7} \mathrm{Be}$ and the release of ${ }^{7} \mathrm{Be}$ from the target material are planned by the end of March 1999.

\section{HIGH RESOLUTION MASS SEPARATOR}

As described in [8], the secondary beams produced in the target-ion source system will go through a magnetic isobaric separator with a nominal resolution power $\mathrm{M} / \Delta \mathrm{M}$ $=20000$.

The EXCYT mass separator will consist of four sections: a pre-separator, two main separation stages at different potential and a transport section towards the tandem. The pre-separator with $\mathrm{M} / \Delta \mathrm{M}=170$ consists of an $18^{\circ}$ bending magnet and a quadruplet of electrostatic quadrupoles placed in a $250 \mathrm{kV}$ platform. Two triplets of electrostatic quadrupoles will guide and focus the beam to a chargeexchange cell.

The first separation stage with $\mathrm{M} / \Delta \mathrm{M}=2000$ is placed on a second $250 \mathrm{kV}$ platform and consists of two bending $77^{\circ}$ and $90^{\circ}$ magnets preceded and followed by a quadruplet of electrostatic quadrupoles. Finally, the second separation stage with $\mathrm{M} / \Delta \mathrm{M}=20000$ consists of two large $90^{\circ}$ bending magnets with surface coils assembled on their pole faces to compensate for misalignment effects. The different potential of the two main stages allows having zero energy dispersion. Consequently the system can be set as an energy achromatic separator to compensate the energy spread of the source.

The transport section consists of three $90^{\circ}$ bending magnets preceded by a quadruplet of electrostatic quadrupoles. The magnets of the separator and of the primary beam line will be delivered in a few weeks; the electrostatic quadrupoles have been designed; one quadruplet has been manufactured and the other elements are under construction.

\section{BEAM DIAGNOSTIC AND SAFETY}

In order to perform the beam diagnostics, besides standard instrumentation we are going to employ tools specially suited for low intensity RIBs:

- A scintillating fibre-based beam profile monitor that can be assembled with either plastic or glass fibre according to the expected intensity range. This device has to be used with high energy beams (after the tandem acceleration) and is planned to be our standard tool for beam handling [9][10].

- A gas detector employing a glass microstrip readout electrode (MSGC), to be used with high energy beams in special cases when the minimum interceptivity is required [11].

- A CsI (Tl) based beam profiler for beams having both high energy and very low intensity. This device is also capable of self-calibration by single particle counting in its lower intensity range. As for the beam identification we are assembling two different complementary devices to be placed respectively before and after the tandem acceleration.

- A beam imaging and identification station based on the detection of the decay products from radioactive ions implanted on an inert tape. This device operating at $200 \mathrm{keV}$ before the tandem acceleration is insensitive to the stable beams.

- A quantitative and qualitative beam analyser, to be used after the acceleration, based on a silicon telescope that detects the beam particles scattered from a thin gold target.

A robot will remove and store the activated target-ion source assembly. The same robot will then place a new assembly. In a similar way, activated parts from the CS (electrostatic deflectors, dees, etc.) will be changed by remote handling. As for the target-ion source assembly, the whole system will be ready by December 1999 while the rest is still under construction.

Gaseous and liquid waste from activated/contaminated areas will be treated before release in the environment. The design for both systems is ready and the relevant mechanical parts will be ordered in a few weeks.

\section{REFERENCES}

[1] G. Ciavola et al., these proceedings and S. Gammino et al., Rev. Sci. Instrum. (in press)

[2] D. Rifuggiato et al.; First years of operation of the LNS Superconducting Cyclotron, Proc. $15^{\text {th }}$ Int. Conf. Cycl. Appl.; Caen, 1998 (in press)

[3] D. Rifuggiato et al.; Axial Injection in the LNS Superconducting Cyclotron, Proc. 15 ${ }^{\text {th }}$ Int. Conf. Cycl. Appl.; Caen, 1998 (in press)

[4] S. L. Snyder; Study and Redesign of the NSCL K500 Injection, Central Region and Phase selection Systems; Ph.D. Thesis, MSU, 1995

[5] J. Bailey et al.; Proc. $13^{\text {th }}$ Int. Conf. Cycl. Appl., Vancouver, (431) 1993

[6] HRIBF News, Edition 7, n 1, Winter Quarter 1999

[7] M. Menna et al.; (in preparation)

[8] G. Ciavola et al.; Design of the EXCYT Radioactive Ion Beam Separator; NIM B 126 (1997) 17

[9]P. Finocchiaro et al.; A Scintillating Fibre-based Profiler for Lowintensity Ion Beams; NIM A 385 (1997) 31

[10] P. Finocchiaro et al.; Low-intensity Ion Beam Profiling with Glass Scintillating Fibres; NIM A 419 (1998) 83

[11] P. Finocchiaro et al.; Particle Detectors for Low Intensity Ion Beam Diagnostics; Proc. $15^{\text {th }}$ Int. Conf. Appl. Acc. Res. Ind.; Denton, 1998 (in press) 